\title{
Antitumor activity of doxorubicine-loaded nanoemulsion against Ehrlich ascites carcinoma-bearing mice
}

\author{
Mayson H Alkhatib ${ }^{1 \star}$, Huda M Alkreathy $^{2}$, Khadijah S Balamash ${ }^{1}$ and Faiza \\ $\mathrm{Abdu}^{3}$ \\ ${ }^{1}$ Department of Biochemistry, Faculty of Science, ${ }^{2}$ Department of Pharmacology, Faculty of Medicine, ${ }^{3}$ Department of Biological \\ Sciences, Faculty of Science, King Abdulaziz University, Jeddah, Saudi Arabia
}

*For correspondence: Email: mhalkhatib@kau.edu.sa; Tel: +966599240526; Fax: + 96626400376

\begin{abstract}
Purpose: To evaluate the antitumor activity of doxorubicine (DOX)-loaded nanoemulsion (NE) on Ehrlich ascites carcinoma (EAC)-bearing Swiss albino mice.

Methods: The mice were divided into five groups $(n=20)$ according to the administered drug. Groups I - $V$ were labeled as negative control (normal), positive control of the untreated EAC bearing mice (EAC control), blank nanoemulsion (BI-NE), DOX-loaded-NE (DOX/LNE) and free DOX (DOX-Sol), respectively. Cardiotoxicity was assessed by measuring changes in body and organ weight, analyzing serum enzymes and lipids, and examining histological changes in heart tissues by light microscopy. In addition, mean survival time (MST), increase in life span (ILS) and survival (S) of the mice were determined.

Results: DOX/LNE group reduced levels of serum enzymes and lowered damage to heart tissues relative to DOX-Sol group. The MST of the DOX/LNE group ( $80 \pm 0.0$ days) was significantly greater than that for DOX-Sol group (34.6 \pm 8.9 days), while ILS of DOX/LNE (265.30 days) was higher than that of DOX-Sol (57.99 days) by 4.6-fold.

Conclusion: Administration of DOX/LNE to EAC-bearing mice improves the efficacy of DOX and reduce its side effects on the heart.
\end{abstract}

Keywords: Doxorubicine, Anti-tumor activity, Mean survival time, Heart histology, Nanoemulsion, Lipid profile

Tropical Journal of Pharmaceutical Research is indexed by Science Citation Index (SciSearch), Scopus, International Pharmaceutical Abstract, Chemical Abstracts, Embase, Index Copernicus, EBSCO, African Index Medicus, JournalSeek, Journal Citation Reports/Science Edition, Directory of Open Access Journals (DOAJ), African Journal Online, Bioline International, Open-J-Gate and Pharmacy Abstracts

\section{INTRODUCTION}

Cancer is a potential fatal disease, characterized by uncontrolled growth and spread of abnormal cells [1]. In spite of the spread of many anticancer drugs, there are still challenges in employing them in cancer therapy because of their poor bioavailability, water insolubility, high toxicity and nonspecific targeting which result in damaging the healthy cells. Doxorubicin (DOX) is one of the most effective anticancer drugs, with a wide scope of activity in human cancers, including acute lymphoblastic leukemia, breast carcinoma, ovarian carcinoma, and hepatocellular carcinoma [2]. However, its clinical application is limited by its harmful side effects, the most significant of which is its cardiotoxicity that can lead to cardiomyopathy and congestive heart failure [3].

A variety of approaches have been reported to minimize the effective chemotherapeutic dose and thereby its side effects. It is possible to devise nanocarriers to deliver anticancer drugs 
which would allow efficient passage and high bioavailability of the anticancer drugs. Nanoemulsions (NE) are one of the promising nanocarriers for many drugs [4]. They are nonequilibrium, heterogeneous systems consisting of two immiscible liquids and mixed surfactants and cosurfactants which facilitate the dispersion of one liquid in the other as droplets with diameters ranging from 1 to $100 \mathrm{~nm}$ [5]. Anticancer drugs loaded in NE systems have greater activity against cancer cells in comparison to other emulsification systems. This is due to the decreased particle size and zeta potential, production of a stable water dispersion, reduced polydispersity index, and greater stability of drug with the NE [6].

In the current study, the anticancer activity of the DOX/LNE on the EAC bearing mice was elaborated.

\section{EXPERIMENTAL}

\section{Materials}

DOX hydrochloride was obtained from Pharmacopeia, California, US. Soya phosphatidylcholine (SPC), sodium oleate (SO), tris (hydroxymethyl) amino methane, polyoxyethyleneglycerol trihydroxystearate 40 (Eumulgin HRE 40, EU) and hydrochloric acid $(\mathrm{HCl})$ were purchased from Leo Chem., S. Puran, Bangalore, India. Cholesterol ( $\mathrm{CHO}$ ) was purchased from Jechno pharmacies BanadurGarh, Haryana, India. 1-Octanol was obtained from AflaAesar $\mathrm{GmbH}$ \& Co KG Karlsruhe, Germany.

\section{Subjects}

Healthy female Swiss albino mice weighing 25 $30 \mathrm{~g}$ were used for the experiment. The animals were housed in large polypropylene cages with utmost of five animals per cage and maintained under standard laboratory conditions (temperature $25 \pm 2{ }^{\circ} \mathrm{C}$ with dark/light cycle $12 / 12 \mathrm{~h}$ ) and provided with standardized pelleted feed and clean drinking water ad libitum. The animals were maintained in accordance with King Abdulaziz University's policy and international guidelines on the care and use of laboratory animals [7]. Ethical approval was obtained from Research Ethics Committee of Faculty of Medicine at King Abdulaziz University (approval ref. no. 189/247/1433). Ehrlich ascites carcinoma (EAC) cells were obtained from American Type Tissue Culture Collection (Manassas, VA, USA).

\section{Preparation of nanoemulsion formulations}

The nanoemulsion (NE) formulation was prepared as described elsewhere [8]. In brief, a $1.25 \% \quad(\mathrm{wt} / \mathrm{wt})$ of the solid surfactants, $\mathrm{EU} / \mathrm{SPC} / \mathrm{SO}$, were mixed at fixed ratio of 3.5:3.0:3.5. Then, a $0.125 \%(w t / w t)$ of the oil phase of $\mathrm{CHO}$ was added slowly to the surfactant mixture until a semisolid phase was formed. After that, a $1 \%$ (wt/wt) of 1-octanol was added dropwise. Finally, the mixture was diluted with the aqueous phase of $97.63 \%$ (wt/wt) of warm Tris- $\mathrm{HCl}$ buffer $(\mathrm{pH} 7.22)$. The mixture was kept in the water bath at $75^{\circ} \mathrm{C}$ for three hours to be clear and transparent and the resulted NE formula was stored at $25{ }^{\circ} \mathrm{C}$. The NE formulas were $\mathrm{BI}-\mathrm{NE}$, DOX/LNE, prepared by solubilizing $2.0 \mathrm{mg}$ of DOX/ kg of mice in $0.2 \mathrm{ml}$ of NE. The DOX-Solution (DOX-Sol) was prepared by dissolving $2.0 \mathrm{mg}$ of DOX in $0.2 \mathrm{ml}$ of distilled water.

\section{In vivo antitumor activity}

\section{Transplantation of tumor and experimental protocol}

The in vivo evaluation of the antitumor activity of different drug formulations was performed by using the regimen of Alkreathy et al [9]. The EAC cells were maintained in the ascitic form by sequential passages in the mice, by means of intraperitoneal (i.p.) transplantation of $2.5 \times 10^{6}$ cells/mouse every 10 days. Ascitic fluid was drawn out from EAC bearing mice at the 8th day following transplantation. Before injection, the viable EAC cells, collected from the peritoneal cavity, were counted by trypan blue exclusion test.

The mice were divided into five groups $(n=20)$ according to the administered drug, at which Group I-V were labeled as negative control (normal), positive control of the untreated EAC bearing mice (EAC control), BI-NE, DOX/LNE and DOX-Sol, respectively. The body weight of all mice was taken before injection with EAC cell. All of the mice in each group were injected i.p. with $2.5 \times 10^{6} \mathrm{EAC}$ cells/mouse at day zero, except group I which was injected with saline solution. After $24 \mathrm{~h}$, animals in Groups III-V were injected with 3 doses of the desired drug, every other day.

On the 15th day, ten mice from each group were fasted for $24 \mathrm{~h}$ and the body weight of each mouse was taken on a digital scale. Then, the blood was collected by a retro orbital plexus method for the estimation of serum biochemical assays. Finally, these animals were sacrificed for 
histology investigation. The rest of the animals in each group were kept for the survival study.

\section{Relative organ weight}

Immediately following sacrifice, the hearts of all of the mice were removed, washed with buffer and dried on blotting paper. Then all hearts were weighed on a digital scale. To compute the organ weight-to-body weight ratio, the post-sacrifice organs (heart) weight (B) from the same animal was divided by the pre-sacrifice body weight for each animal $(A)$.

\section{Determination of biochemical parameters}

For biochemical assays, the blood samples were allowed to clot and the serum was separated by centrifugation at 5000 RPM for 10 min. Serum was utilized for the estimation of cardiac enzymes (creatine phosphokinase (CK) and lactate dehydrogenase (LDH)) and the serum lipid profile (cholesterol (CHO), high density lipoprotein (HDL) and triglyceride (TG)). The biochemical assays were performed according to the standard kit methods using fully Automated COBAS $\AA 8000$ modular analyzer series in the King Fahd Armed Forces Hospital-Jeddah, Saudi Arabia.

\section{Histological studies}

The collected heart tissues were fixed in $10 \%$ neutral buffered formalin (4\% formaldehyde in phosphate buffered saline). Thereafter, the tissues were incrementally dehydrated by increasing concentrations of alcohol and then stained with hematoxylin and eosin $(H \& E)$, followed by microscopic examination.

\section{Survival study}

The observation period of the survival study began in the first weeks of age until the study ended at the twelfth week of age. The MST, \% ILS and \% S of each group containing ten mice were monitored by recording the daily mortality for 80 days. ILS were calculated using Eq 1 [10].

$$
\text { ILS }(\%)=\{(\mathrm{St} / \mathrm{Sc})-1\} 100
$$

where St and Sc are the mean survival time of treated and control groups, respectively.

\section{Statistical analysis}

All values were expressed as mean \pm standard deviation (SD; $n=10)$. Statistical analysis was performed with one-way analysis of variance (ANOVA) test and independent sample t-test using MegaStat Excel (version 10.3, Butler University). The significant differences between the tested samples were classified according to the $p$-value. If $p<0.001$, the difference was considered very highly significant $\left(^{* * *}\right) ; p<0.01$ was considered highly significant $\left({ }^{* \star}\right)$; the difference was considered significant at $p<0.05$ $\left({ }^{*}\right)$.

\section{RESULTS}

\section{Body weight change and relative organ weight}

As displayed in Figure 1A, there was a very high significant increase in the body weight changes of EAC control and BI-NE groups compared with the normal group $(P$-value $<0.001)$ whereas the enhancement in the body weight change was reduced in the DOX/LNE group $(p<0.05)$. On the other hand, there was a significant decrease in the body weight change in the DOX-Sol group $(p<0.05)$. Studying the effect of the drug formulations on the heart weight relative to the body weight revealed that the mice treated with DOX-Sol, DOX/LNE and BI-NE showed no significant difference from the normal group in heart-to-body weight ratio, whereas EAC control group showed a significant decrease in the heartto-body weight ratio (Figure 1B).

\section{Cardiac enzymes and lipid profile}

The effect of the drug formulations on the heart enzymes and lipid profile is exhibited in Table 1. The CK levels in the EAC control and BI-NE groups were comparable with the normal group. In spite of the significant increase in the CK levels in both of DOX-Sol and DOX/LNE relative to the normal group, the CK level of DOX/LNE group was significantly less than DOX-Sol group. Nevertheless, the CK levels in all of the groups were within the standard range of the enzyme in the mice (50-680 IU/I). Furthermore, all treated groups showed a significant increase in the LDH levels when compared to the normal group. However, the increase in the LDH levels in all of the groups were within the standard range of the albino mice, which is $(85-750 \mathrm{IU} / \mathrm{l})$, except DOX-Sol group which had elevated levels of LDH.

Regarding the lipid profile, there were no significant changes in $\mathrm{CHO}$ level between the normal group and the other treated groups except DOX-Sol group which had reduced amount of $\mathrm{CHO}$. On the other hand, all treated groups did not show any considerable difference 

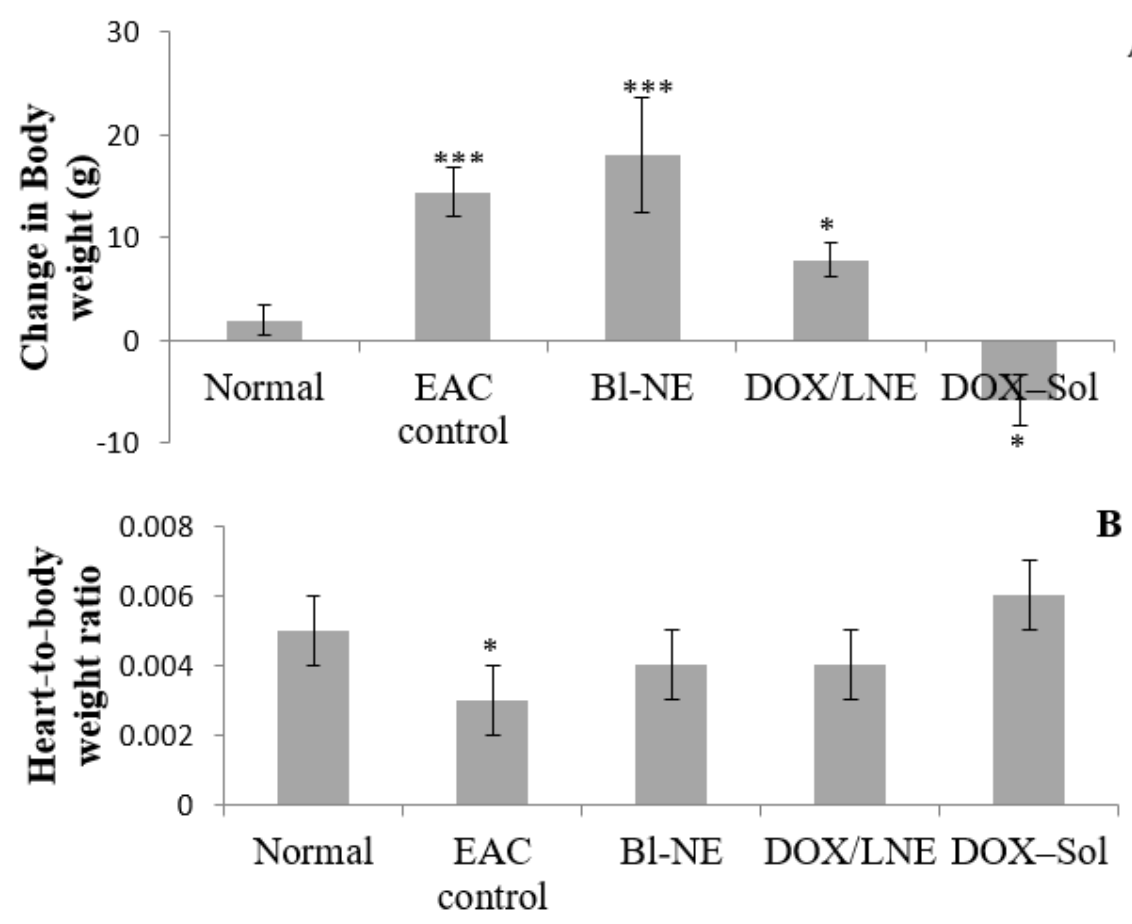

Figure 1: The effect of the drug formulations on the A) body weight change and B) heart-to-body weight ratio of EAC bearing mice. The significant differences between the treated and the normal groups were classified statistically according to the $p$-value. If the $p<0.001$, the difference was considered very highly significant $\left({ }^{* * *}\right)$ while if $0.001<p<0.01$ was considered highly significant $\left(^{* *}\right)$. If the $p<0.05$, the difference was considered significant $\left({ }^{*}\right)$

Table 1: Effect of drug formulations on the cardiac enzymes and lipid profile in EAC-bearing mice

\begin{tabular}{|c|c|c|c|c|c|}
\hline Group & $\overline{C K}$ & LDH & $\mathrm{CHO}$ & HDL & $\overline{T G}$ \\
\hline Normal & $136.63 \pm 89.28$ & $163.80 \pm 145.85$ & $28.00 \pm 3.44$ & $21.03 \pm 6.21$ & $12.80 \pm 0.62$ \\
\hline EAC Control & $206.88 \pm 76.33$ & $589.66 \pm 42.71$ & $24.70 \pm 4.52$ & $14.00 \pm 2.52$ & $18.23 \pm 9.44$ \\
\hline $\mathrm{BI}-\mathrm{NE}$ & $182.33 \pm 114.94$ & $758.33 \pm 79.09$ & $28.77 \pm 3.41$ & $17.33 \pm 4.85$ & $16.00 \pm 6.94$ \\
\hline DOX/LNE & $423.44 \pm 7.12^{x \times x}$ & $594.66 \pm 303.00^{\pi \pi}$ & $32.30 \pm 1.60$ & $24.95 \pm 3.55$ & $15.65 \pm 3.85$ \\
\hline DOX-Sol & $603.66 \pm 21.68^{\pi \times \pi}$ & $1074.44 \pm 36.71^{\pi \times \pi}$ & $18.00 \pm 1.00^{\pi \pi}$ & $15.47 \pm 0.45$ & $5.90 \pm 0.30$ \\
\hline
\end{tabular}

Significant differences between the treated and the normal groups were classified statistically according to the $p$ value. If $p<0.001$, the difference was considered very highly significant $\left({ }^{* * *}\right)$ while if $0.001<p<0.01$ was considered highly significant $\left({ }^{* *}\right)$. If $p<0.05$, the difference was considered significant $\left(^{*}\right)$

in HDL and TG levels when compared to the normal group.

\section{Cardiac histopathology}

The histopathological examination of the heart tissues of mice in the EAC control group showed normal architectures as no inflammatory cell infiltration was seen (Figure 2A). The tissue of the mice treated with BI-NE showed normal myocardium morphology almost similar to the EAC control group except some nuclei have endured karyolysis, which is known as the complete dissolution of the chromatin of a dying cell due to enzymatic degradation by endonucleases, while others were pyknotic, which is the irreversible condensation of chromatin in the nucleus of a cell undergoing necrosis or apoptosis followed by fragmentation of the nucleus (Figure 2B). Mice treated with DOX-Sol showed damages in the heart tissues, including the loss of myofibrils (myofibrillar fragmentation), congestion of blood vessels, a stimulation of cytoplasmic vacuolization and some nuclei enduring karyolysis and pyknosis (Figure 2C). DOX-LNE group had a significant reduction in the severity of myocardial degeneration that was observed as mild disruption of cardiac muscle fibers and reduced cytoplasmic vacuolization (Figure 2D). 

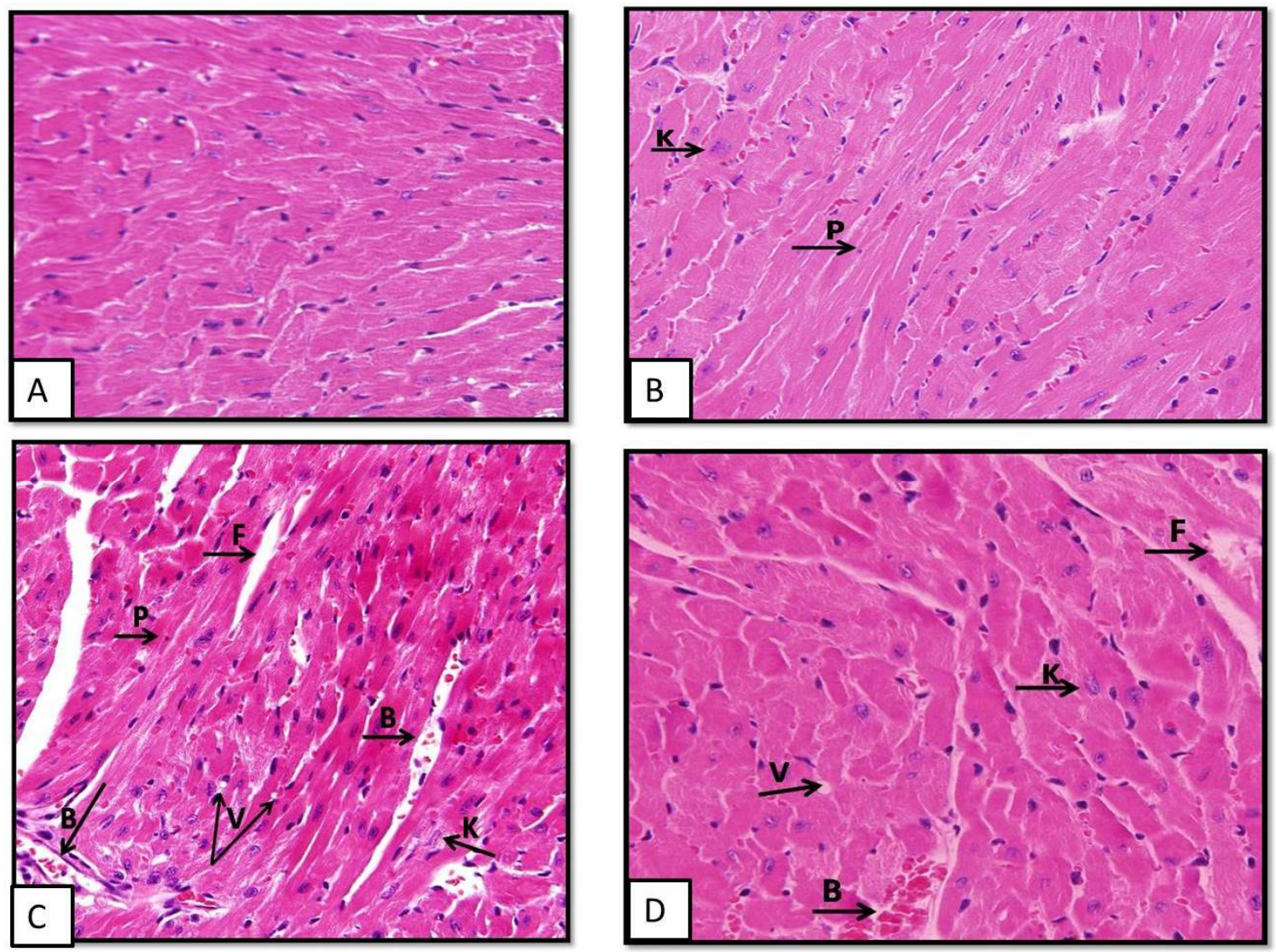

Figure 2: Photomicrographs of the myocardium of A) EAC control group, showing normal morphology $B$ ) BI-NE group, showing normal morphology almost similar to normal group except some cardiomyocytes endured karyolysis $(\mathrm{K})$ while others were pyknotic $(\mathrm{P}), \mathrm{C})$ DOX-Sol group, showing damage in cardiomyscytes as indicated by increased cytoplasmic vacuolization (V), myofibrillar fragmentation ( $F$ ) and congestion of blood vessels (B), and D) DOX/LNE group, revealing slight alteration in the morphology of the cardiomyocytes and considerably less cellular vacuolization and myofibrillar damage. $\mathrm{H} \& \mathrm{E} \times 400$

Table 2: Effect of drug formulations on the MST, \% ILS and \% S.

\begin{tabular}{lccc}
\hline Group & MST (days) & ILS (\%) & S (\%) \\
\hline Normal & $80 \pm 0$ & \multicolumn{1}{c}{100} \\
EAC Control & $21.9 \pm 1.729^{\cdots \cdots}$ & - & 0 \\
BI-NE & $22.5 \pm 2.224^{\cdots}$ & 2.740 & 0 \\
DOX/LNE & $80 \pm 0$ & 265.297 & 100 \\
DOX-Sol. & $\mathbf{3 4 . 6 \pm 8 . 8 7 7 ^ { \cdots \cdots }}$ & $\mathbf{5 7 . 9 9 1}$ & $\mathbf{0}$ \\
\hline
\end{tabular}

Significant differences between the treated and the normal groups were classified statistically according to the Pvalue. If $p<0.001$, the difference was considered very highly significant $\left({ }^{* * *}\right)$ while if $0.001<p<0.01$ was considered highly significant $\left(^{* *}\right)$. If $p<0.05$, the difference was considered significant $\left(^{*}\right)$

\section{Survival}

The effect of the drug formulations on the MST of EAC-bearing mice is illustrated in Table 2. Interestingly, the MST and \% S of the DOX/LNE group was equivalent to the MST and \% S of the normal group which was very highly significantly greater than the MST and \% $S$ of the EAC control, BI-NE and DOX-Sol groups. The \% ILS of DOX/LNE was longer than the \% ILS of DOXSol by 4.6 folds.

\section{DISCUSSION}

The present study was carried out to evaluate the antitumor effect of the DOX-loaded-NE in one of the most devastating experimental tumor models, the EAC bearing mice. Because of their biocompatibility, NEs have been widely used in the medical field for delivery and controlled release of the hydrophobic anticancer drugs because they present a very large surface area and good long-term stability. As expected in the 
EAC control group, a significant increase in body weight was observed [10]. However, animals treated with DOX-Sol showed a further significant decrease in the body weight indicating more reduction in the i.p. tumor burden. This result was similar to several studies which demonstrated that the use of DOX leads to a significant drop in the body weight of rats $[11,12]$. In 2013, Lv et al [13] proved that DOX loaded in nanoparticles exhibited higher antitumor efficacy on A549 lung-tumor-bearing mice compared with free DOX at the same dose. Moreover, from our experimental groups, we found that DOX/LNE group had a significant decrease in the body weight compared to the EAC control group.

The reliable criterion for judging the value of any anticancer drug is the prolongation of life span of the tumor bearing animal [14]. The MST, \% ILS and $\% S$ of EAC bearing mice treated with DOX/LNE were significantly longer than EAC bearing mice treated with DOX-Sol. This study is in agreement with Xiong et al [15] study, which showed that the life span of animals bearing MDA-435/LCC6WT (human breast cancer cell line) and treated with polymeric nanocarriers of DOX, was significantly longer than animals treated with free DOX. Additionally, Hao et al [16] demonstrated that nanoparticles can decrease the toxicity of DOX that result in a significant increase of the average lifetime in comparison with the free DOX.

In the present work, heart weight-to-body weight ratios were assessed in order to evaluate the toxicity of the drug formulas on the whole organ. Mice treated with drug formulations that contain 2 $\mathrm{mg} / \mathrm{kg}$ DOX did not show any significant change in heart weight-to-body weight ratio compared to the normal group. However, when rats were given three i.p injections of $5 \mathrm{mg} / \mathrm{kg}$ DOX, a significant less mean ratio of heart weight to body weight against the control animals was reported [17].

DOX is a very potent antitumor antibiotic. Its use is severely limited for its cardiotoxicity, which have been documented in a variety of animal models [12]. During malignancy, the tumor marker enzymatic changes reflect overall changes in metabolism and they are increasingly appreciated as critical determinants of tumor cell behavior with sensitivity and specificity [18].

The results of the present study indicate that mice treated with DOX-Sol had a significant increase in LDH and CK levels, important markers of cardiac injury, especially during clinical follow-up of DOX therapy. In agreement with our findings, Yagmurca et al [19] demonstrated similar elevations in cardiac enzymes activities in rats following challenge with a single cumulative dose of DOX. Generation of free radicals by DOX caused extensive damage in the myocardium, which result in increased membrane permeability and hence leads to leakage of $\mathrm{LDH}$ and CK enzymes. On the other hand, administering DOX/LNE into the EAC bearing mice reduced the enhancement in the cardiac enzymes relative to the DOX-Sol. Park et al [20] proved that DOX-loadednanoparticle decreased the observed cardiac toxicity by decreasing CK level compared to the DOX treated group.

In the present study, it has been found that mice treated with Dox-Sol had significantly decreased levels in $\mathrm{CHO}$ compared to normal group. The levels of TG and HDL in mice were not affected by the different treated drug formulas. In contrast, rat treated with DOX at a total cumulative dose of $15 \mathrm{mg} / \mathrm{kg}$ through i.p showed that DOX interferes with metabolism and biosynthesis of lipids led to significantly increased levels of TG, $\mathrm{CHO}$ and decreased level of HDL [21].

According to the light microscopy images in the present study, heart sections of mice treated with DOX-Sol showed massive pathological changes compared to the normal architecture observed in the EAC control group. As observed in the heart tissue, the damages induced by DOX-Sol include the loss of myofibrils, congestion of blood vessels, stimulation in the cytoplasmic vacuolization and some nuclei enduring karyolysis and pyknosis. The histopathological changes observed in the DOX-Sol group were similar to those previously reported [22-24]. As noted from the histological reports, the heart myocytes degeneration could have resulted in the leakage of enzymes into the blood stream. Unlike DOX-Sol group, the heart tissue of DOX/LNE group was similar to the EAC control group sections in showing a normal tissue architecture. This result indicated that loading DOX in NE formulation reduced the damages and toxicity of DOX on the heart tissues.

\section{CONCLUSION}

The main goal of the current study was to assess the anticancer activity of DOX-loaded NE in vivo. Loading DOX into NE considerably improves its efficacy while reducing its cardiotoxicity. These findings suggest that $N E$ is a promising nanocarrier for DOX. However, studies using other animals are required to confirm these findings and evaluate the possible toxicological effects of this new formulation in human patients. 


\section{ACKNOWLEDGEMENT}

This project was funded by the Deanship of Scientific Research (DSR) at King Abdulaziz University, Jeddah (grant no. 189/247/1433). The authors, acknowledge with thanks the DSR for technical and financial support.

\section{CONFLICT OF INTEREST}

The authors declare no conflict of interest associated with this work.

\section{CONTRIBUTION OF AUTHORS}

We declare that this work was done by the authors named in this article and all liabilities pertaining to claims relating to the content of this article will be borne by the authors. MHA conceived this study. MHA, HMA, KSB and FA designed the experiments and analyzed the data while MHA carried out the statistical analysis the data and wrote the manuscript. All authors read and approved the manuscript for publication.

\section{REFERENCES}

1. Senthilkumar K, Manivasagan P, Venkatesan J, Kim SK. Brown seaweed fucoidan: biological activity and apoptosis, growth signaling mechanism in cancer. Int $J$ Biol Macromol 2013; 60: 366-374.

2. Zheng J, Lee HC, Bin Sattar MM, Huang Y, Bian JS. Cardioprotective effects of epigallocatechin-3-gallate against doxorubicin-induced cardiomyocyte injury. Eur $J$ Pharmacol 2011; 652 (1-3): 82-88.

3. Ibsen S, Zahavy E, Wrasdilo $W$, Berns $M$, Chan $M$, Esener S. A novel doxorubicin prodrug with controllable photolysis activation for cancer chemotherapy. Pharm Res 2010; 27 (9): 1848-1860.

4. Shah $P$, Bhalodia $D$, Shelat $P$. Nanoemulsion: $A$ pharmaceutical review. Syst Rev Pharm 2010; 1 (1): 2432.

5. Mason TG, Wilking JN, Meleson K, Chang CB, Graves SM. Nanoemulsions: formation, structure, and physical properties J. Phys.: Condens. Matter 2006; 18 (41): R635-R666.

6. Alkhatib MH, Albishi HM, Mahassni SH. Impact of Nanoparticles on Cancer Therapy. Trop J Pharm Res 2012; 11 (6): 1001-1011.

7. National Research Council of The National Academy of Sciencies. Guide for the Care and Use of Laboratory Animals: Eight Edition. Washington, D.C.: The National Academies Press; 2010.

8. Alkhatib $\mathrm{MH}$, AlBishi HM. In vitro evaluation of antitumor activity of doxorubicin-loaded nanoemulsion in MCF-7 human breast cancer cells. J Nanopart Res 2013; 15 (3): 1-15.
9. Alkreathy $H$, Damanhouri ZA, Ahmed $N$, Slevin $M$, Osman AM. Mechanisms of cardioprotective effect of aged garlic extract against doxorubicin-induced cardiotoxicity. Integr Cancer Ther 2012; 11: 364-370

10. Raju AB, Ravindranath A, Kalpana G. Antitumor activity of Diospyros peregrina on Ehrlich ascites carcinoma in mice. J Sci Res 2011; 3(2): 413-419.

11. Injac $R$, Perse $M$, Cerne $M$, Potocnik $N$, Radic $N$, Govedarica $B$, Strukelj $B$. Protective effects of fullerenol C60(OH)24 against doxorubicin-induced cardiotoxicity and hepatotoxicity in rats with colorectal cancer. Biomater 2009; 30 (6): 1184-1196.

12. Rašković A, Stilinović $N$, Kolarović J, Vasović $V$, Vukmirović S, Mikov M. The protective effects of silymarin against doxorubicin-induced cardiotoxicity and hepatotoxicity in rats. Molecule 2011; 16(10): 86018613.

13. Lv S, Li M, Tang $Z$, Song $W$, Sun $H$, Liu $H$, Chen $X$. Doxorubicin-loaded amphiphilic polypeptide-based nanoparticles as an efficient drug delivery system for cancer therapy. Acta Biomater 2013; 9 (12): 9330-9342.

14. Haldar PK, Kar B, Bala A, Bhattacharya S, Mazumder UK. Antitumor activity of Sansevieria roxburghiana rhizome against Ehrlich ascites carcinoma in mice. Pharm Biol 2010; 48 (12): 1337-1343.

15. Xiong XB, Ma Z, Lai $R$, Lavasanifar $A$. The therapeutic response to multifunctional polymeric nano-conjugates in the targeted cellular and subcellular delivery of doxorubicin. Biomat 2010; 31(4): 757-768.

16. Hao $H$, Ma $Q$, Huang $C$, He F, Yao P. Preparation, characterization, and in vivo evaluation of doxorubicin loaded BSA nanoparticles with folic acid modified dextran surface. Int J Pharm 2013; 444 (1): 77-84.

17. Ramadan $W$. The protective effect of $\alpha$-lipoic acid in doxorubicin induced cardiotoxicity in rats [dissertation]. [Texas]: Baylor University; 2008 Aug. p 67.

18. Shivanandappab T. Antitumor Effect of Decalepis Hamiltonii Root Extract against Ehrlich Ascites Tumor in Mice. Int J Pharm Bio Sci 2013; 4(3): 88-96.

19. Yagmurca M, Fadillioglu E, Erdogan $H$, Ucar $M$, Sogut $S$, Irmak MK. Erdosteine prevents doxorubicin-induced cardiotoxicity in rats. Pharmacol Res 2003; 48 (4): 377382.

20. Park J, Fong PM, Lu J, Russell KS, Booth CJ, Saltzman WM, Fahmy TM. PEGylated PLGA nanoparticles for the improved delivery of doxorubicin. Nanomed 2009; 5 (4): 410-418.

21. Chennuru A, Saleem MT. Antioxidant, lipid lowering, and membrane stabilization effect of sesamol against doxorubicin-induced cardiomyopathy in experimental rats. Biomed Res Int 2013; 2013: 934-239

22. Divakaran SA, Nai CK. Amelioration of doxorubicin induced cardiotoxicity in tumor bearing mice by ferulic acid: a mechanistic study at cellular and biochemical level. Int J Tumor Therap 2012; 1 (2): 6-13.

23. Ahmed MA. The Protective Effect of Ginger (Zingiber Officinale) Against Adriamycin-Induced Hepatotoxicity in 
Alkhatib et al

Rats: Histological Study. Life Sci J 2013; 10 (1): 1412 1422.

24. Salouege I, Ali RB, Saïd DB, Elkadri N, Kourda N, Lakhal $M$, Klouz A. Means of evaluation and protection from doxorubicin-induced cardiotoxicity and hepatotoxicity in rats. J Cancer Res Ther 2014; 10 (2): 274-278. 